Supporting Information for:

\title{
Improved Settling Velocity for Microplastic Fibers: A New Shape-dependent Drag Model
}

Jiaqi Zhang ${ }^{1}$ and Clarence Edward Choi ${ }^{*}$

*Corresponding author, email: cechoi@hku.hk

1. Department of Civil Engineering, The University of Hong Kong.

\section{Contents}

SI1: Details of the experiments of microplastic fibers settling in quiescent water S03

SI2: Strategy for developing the new drag model. S08

SI3: Morphology characterisation of microplastics. S09

SI4: Evaluation of Aschenbrenner shape factor with additional datasets S11

SI5: Assessment of extracted data S13

SI6: Evaluation of the new drag model with estimated fiber volume based on the experiment data produced in this study .$S 14$

SI7: Applicability of the new drag model to weathered microplastics .$S 15$

SI8: Applicability of the new drag model to predictions of terminal rising velocities of virgin microplastics

References .520 


\section{List of Figures}

Figure S1: Details of the fiber settling experiments .............................................S04

Figure S2: Measured velocity time histories of the fibers .....................................S06

Figure S3: Comparison of terminal settling velocities measured in the repeated tests

Figure S4: The derivation procedure of the newly-proposed drag model ................S09

Figure S5: Microplastic classification based on elongation and flatness .................S08

Figure S6: Comparison of performances of Aschenbrenner shape factor and Corey shape factors on distinguishing fibrous and non-fibrous microplastics

Figure S7: Evaluation of newly-proposed model (Eq.6) with the estimated fiber volume provided by experiments conducted in this study

Figure S8: Comparison of performances of drag models proposed by this study and by Waldschlager et al. ${ }^{2}$ on predicting terminal settling/rising velocities of weathered microplastics S15

Figure S9. Performances of drag models proposed by this study and by Waldschlager et al. $^{2}$ on predicting terminal rising velocities of virgin microplastics S17

\section{List of Tables}

Table S1: Summary of properties of fluid and microplastic fibers $\mathrm{S} 03$

Table S2: Summary of measured terminal settling velocities of microplastic fibers S05

Table S3: Comparison between reported and recalculated prediction accuracy S13 


\section{SI1: Details of the experiments of microplastic fibers settling in quiescent water}

Unique experiments were carried out to provide a new and independent dataset to evaluate the newly-proposed drag model (Eq. 6).

\section{(1) Materials and methods}

Materials: The microplastic fibers used in this study were cut from commercial polyester threads that are generally used in the textile industry (Figure S1a). The fiber density was measured by using the titration method ${ }^{1}$, which has been widely used in the literature to measure the density of microplastics. ${ }^{2,3}$ The length and diameter of each fiber were measured based on images captured by using a digital microscope. Table S1 gives a summary of the physical properties of the microplastic fibers tested.

Table S1 Summary of properties of fluid and microplastic fibers*

\begin{tabular}{ccccc}
\hline Test ID & $\begin{array}{c}\text { Volume } \\
\boldsymbol{V}\left[\mathrm{m}^{3}\right]\end{array}$ & $\begin{array}{c}\text { Longest length } \\
\boldsymbol{D}_{\mathbf{L}}[\mathrm{m}]\end{array}$ & $\begin{array}{c}\text { Intermediate length } \\
\boldsymbol{D}_{\mathbf{I}}[\mathrm{m}]\end{array}$ & $\begin{array}{c}\text { Shortest length } \\
\boldsymbol{D}_{\mathbf{S}}[\mathrm{m}]\end{array}$ \\
\hline F01 & $7.35 \times 10^{-10}$ & $5.0 \times 10^{-3}$ & $4.5 \times 10^{-4}$ & $4.5 \times 10^{-4}$ \\
F02 & $9.04 \times 10^{-10}$ & $5.0 \times 10^{-3}$ & $5.5 \times 10^{-4}$ & $5.5 \times 10^{-4}$ \\
F03 & $1.10 \times 10^{-9}$ & $5.0 \times 10^{-3}$ & $6.5 \times 10^{-4}$ & $6.5 \times 10^{-4}$ \\
F04 & $2.80 \times 10^{-9}$ & $5.0 \times 10^{-3}$ & $8.5 \times 10^{-4}$ & $8.5 \times 10^{-4}$ \\
F05 & $1.29 \times 10^{-9}$ & $1.0 \times 10^{-2}$ & $4.5 \times 10^{-4}$ & $4.5 \times 10^{-4}$ \\
F06 & $2.48 \times 10^{-9}$ & $1.0 \times 10^{-2}$ & $5.5 \times 10^{-4}$ & $5.5 \times 10^{-4}$ \\
F07 & $3.88 \times 10^{-9}$ & $1.0 \times 10^{-2}$ & $6.5 \times 10^{-4}$ & $6.5 \times 10^{-4}$ \\
F08 & $8.18 \times 10^{-9}$ & $1.0 \times 10^{-2}$ & $8.5 \times 10^{-4}$ & $8.5 \times 10^{-4}$ \\
F09 & $1.62 \times 10^{-9}$ & $1.5 \times 10^{-2}$ & $4.5 \times 10^{-4}$ & $4.5 \times 10^{-4}$ \\
F10 & $3.15 \times 10^{-9}$ & $1.5 \times 10^{-2}$ & $5.5 \times 10^{-4}$ & $5.5 \times 10^{-4}$ \\
F11 & $6.04 \times 10^{-9}$ & $1.5 \times 10^{-2}$ & $6.5 \times 10^{-4}$ & $6.5 \times 10^{-4}$ \\
F12 & $1.06 \times 10^{-9}$ & $1.5 \times 10^{-2}$ & $8.5 \times 10^{-4}$ & $8.5 \times 10^{-4}$ \\
\hline
\end{tabular}

* Microplastic fiber density $\left(\rho_{\mathrm{m}}\right)$ is $1308 \mathrm{~kg} / \mathrm{m}^{3}$.

Experimental Setup: An acrylic column with a square cross-section of $100 \times 100 \mathrm{~mm}$ and a height of $270 \mathrm{~mm}$ (Figure S1b) was used to measure the terminal settling velocities of microplastic fibers. The column was filled with distilled water. The water density and viscosity are $1000 \mathrm{~kg} / \mathrm{m}^{3}$ and $1.0 \times 10^{-6} \mathrm{~m}^{2} / \mathrm{s}$, respectively. Prior to each test, fibers were submerged in a glass dish with distilled water to minimise the effects of static surface charge on the settling behavior. ${ }^{3}$ Afterwards, each fiber was held about 10 
$\mathrm{mm}$ below the surface of the water at the center of the column by using a tweezer and allowed to settle under the influence of gravity.

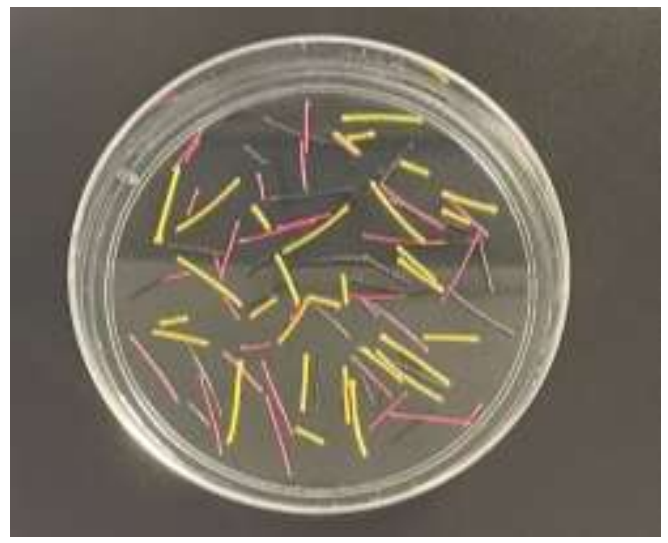

(a)

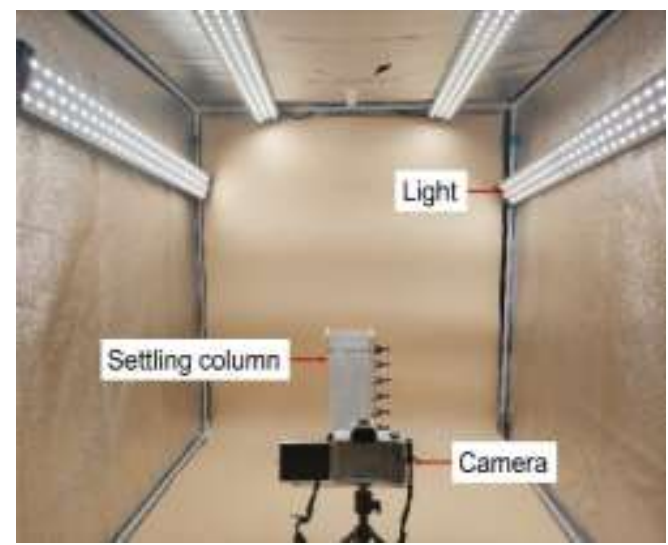

(b)

Figure S1. Details of the fiber settling experiments. (a) Microplastic fibers. Prior to testing, the fibers were submerged in a glass dish filled with distilled water to minimise the effects of static surface charge on the fiber settling behavior; (b) experimental setup.

Velocity measurement: The settling process for each fiber was recorded by using a digital camera with a resolution of $1920 \times 1080$ and a frame rate of $50 \mathrm{fps}$. Several nonpulsating lights were used to illuminate the column (Figure S1b) to enhance imaging. From these videos, a sequence of still images of the settling kinematics was extracted at a time interval of $1 / 50 \mathrm{~s}$ by using MATLAB. Based on the sequential images, the settling distance of each fiber within a known time interval (i.e., 1/50 s) was measured by using the image measurement software, ImageJ. Consequently, the time-varying velocity of each fiber was deduced. The terminal settling velocity of each fiber was extracted from the velocity time history. For each fiber, the terminal settling velocity was taken as the average of three repeated tests. The measured velocities are summarized in Table S2. 
Table S2 Summary of measured terminal settling velocities of microplastic fibers

\begin{tabular}{ccccc}
\hline Test ID & $\begin{array}{c}\text { First test } \\
{[\mathrm{mm} / \mathrm{s}]}\end{array}$ & $\begin{array}{c}\text { Second test } \\
{[\mathrm{mm} / \mathrm{s}]}\end{array}$ & $\begin{array}{c}\text { Third test } \\
{[\mathrm{mm} / \mathrm{s}]}\end{array}$ & $\begin{array}{c}\text { Average velocity } \\
{[\mathrm{mm} / \mathrm{s}]}\end{array}$ \\
\hline F01 & 18.09 & 19.18 & 19.28 & 18.85 \\
F02 & 24.68 & 24.33 & 25.41 & 24.81 \\
F03 & 30.23 & 32.05 & 31.37 & 31.22 \\
F04 & 33.62 & 34.79 & 35.83 & 34.75 \\
F05 & 21.52 & 23.92 & 24.28 & 23.24 \\
F06 & 27.32 & 28.95 & 28.5 & 28.26 \\
F07 & 31.66 & 30.7 & 34.35 & 32.24 \\
F08 & 36.43 & 36.38 & 36.67 & 36.49 \\
F09 & 20.85 & 20.83 & 23.54 & 21.74 \\
F10 & 27.22 & 28.55 & 28.92 & 28.23 \\
F11 & 34.16 & 32.72 & 34.5 & 33.79 \\
F12 & 40.05 & 40.00 & 38.15 & 39.40 \\
\hline
\end{tabular}

\section{(2) Verification that microplastic fibers have reached terminal settling velocities}

Figure S2 shows the real-time settling velocities of the smallest and largest fibers used in the experiments.

It can be observed that the settling velocities of the fibers reach a relatively stable velocity after a stretch of acceleration. Despite some fluctuations in velocity, the relative differences between the maximum and minimum velocities are smaller than $5 \%$ for all tests. Thus, the average values of these relatively stable velocities can be used as the terminal settling velocities of fibers. ${ }^{2}$ 


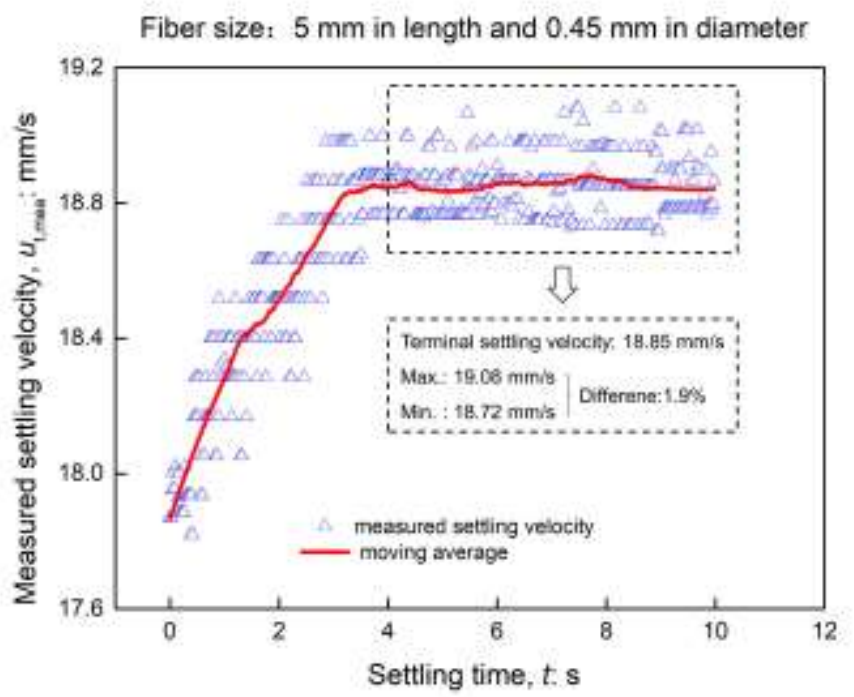

(a)

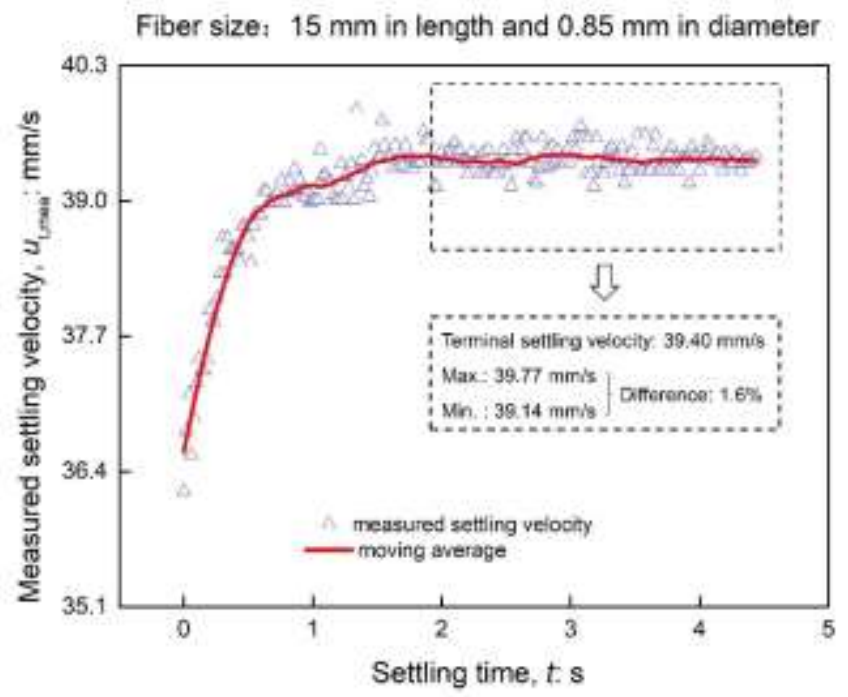

(b)

Figure S2. Measured velocity time histories of the fibers. Measurements are taken (i.e., $t=0$ ) when the fiber is $70 \mathrm{~mm}$ below the water surface. The difference means the relative difference between velocities and is calculated as $\frac{\text { maximum velcocity-minimum velcotiy }}{\text { minimum velociy }} \times 100 \%$.(a) smallest fiber: $5 \mathrm{~mm}$ in length and $0.45 \mathrm{~mm}$ in diameter; (b) largest fiber: $15 \mathrm{~mm}$ in length and $0.85 \mathrm{~mm}$ in diameter. 


\section{(3) Reproducibility of fiber settling experiment}

Figure S3 shows a comparison of the results of three repeated tests for all fibers tested. It can be observed that the tests are repeatable, indicating the high reliability of the terminal settling velocities measured in these experiments.

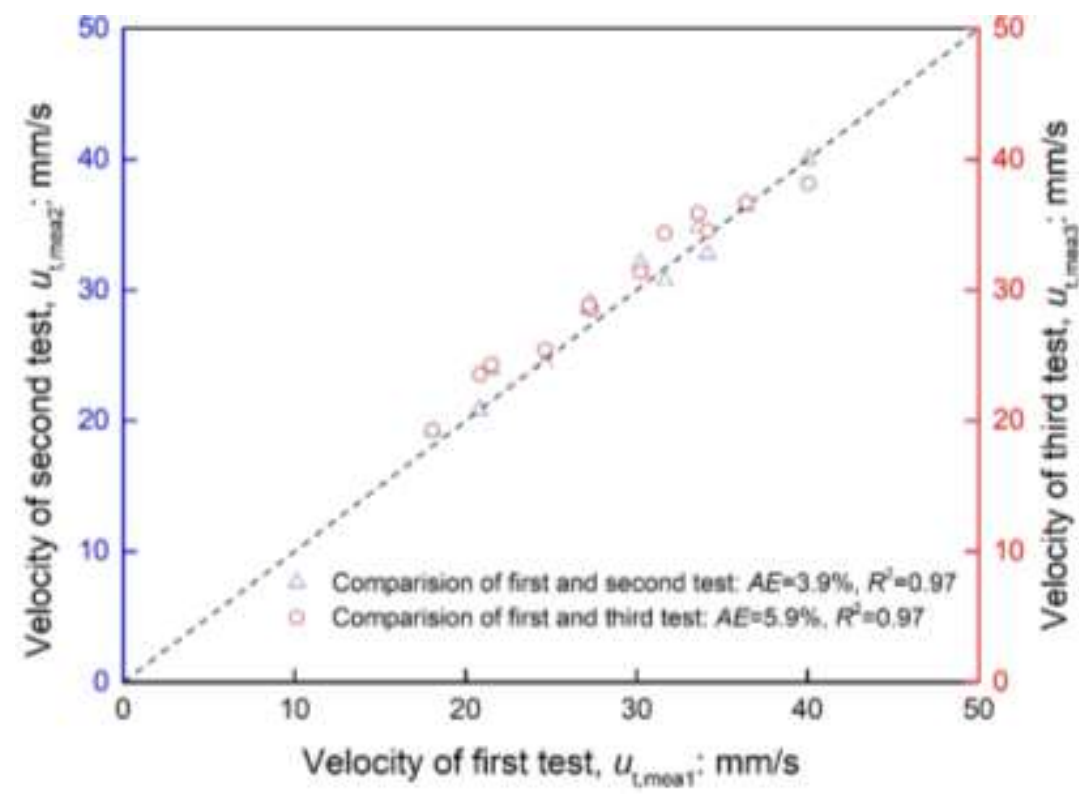

Figure S3. Comparison of terminal settling velocities measured in the repeated tests. $A E$ is the average relative error, and $R^{2}$ denotes the coefficient of determination. The black dashed line indicates that the velocity measured from the second or third test is the same as the one measured from the first test." 


\section{SI2: Strategy for developing the new model}

Figure S4 illustrates the derivation procedure of the newly-proposed drag model (Eq.

$6)$.

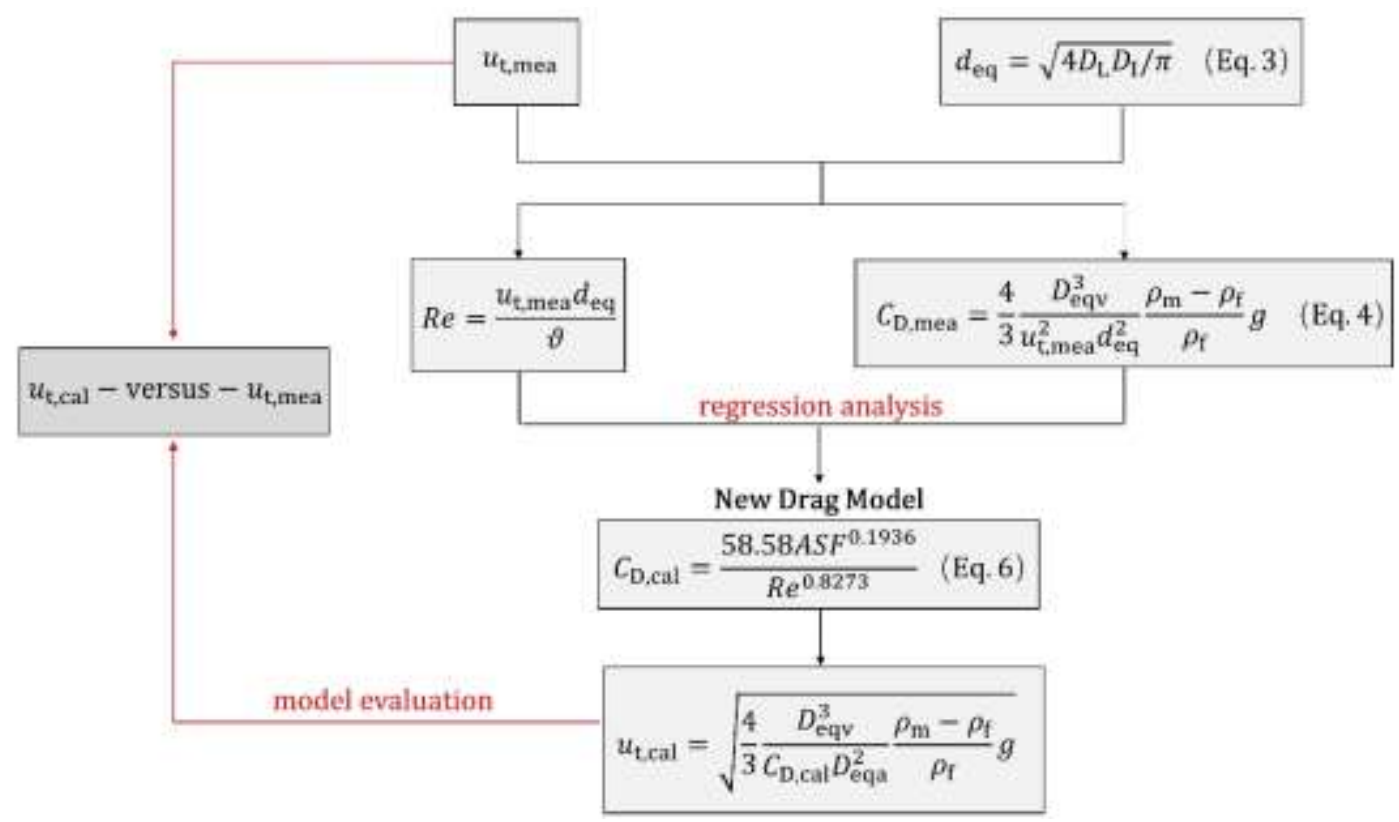

Figure S4. The derivation procedure of the newly-proposed drag model (Eq. 6), where $u_{\mathrm{t} \text {,mea }}$ is the measured velocities of microplastics, $C_{\mathrm{D} \text {,mea }}$ is the measured drag coefficient and is obtained by combining the measured velocities and the expression of drag coefficient proposed in this study (Eq.4), $C_{\mathrm{D}, \text { cal }}$ and $u_{\mathrm{t}, \mathrm{cal}}$ are the calculated drag coefficient and calculated velocity, respectively, which are obtained by using the newly newly-proposed drag model (Eq.6), Re is the Reynolds number. $A r$ is the Archimedes number. $d_{\mathrm{eq}}$ is the equivalent diameter which is calculated by using Eq.3. $\rho_{\mathrm{m}} D_{\mathrm{L}}$, and $D_{\mathrm{I}}$ are the density, longest length, and intermediate length of a microplastic, respectively, and $\rho_{\mathrm{f}}$ and $\vartheta$ are the density and kinematic viscosity of the fluid, respectively. 


\section{SI3: Morphology characterization of microplastics}

Figure S5 categories the morphologies of different microplastic particles reported by

Melkebeke et al. ${ }^{3}$ using the indices of elongation $\left(e=D_{\mathrm{I}} / D_{\mathrm{L}}\right)$ and flatness $\left(f=D_{\mathrm{S}} / D_{\mathrm{I}}\right)$.

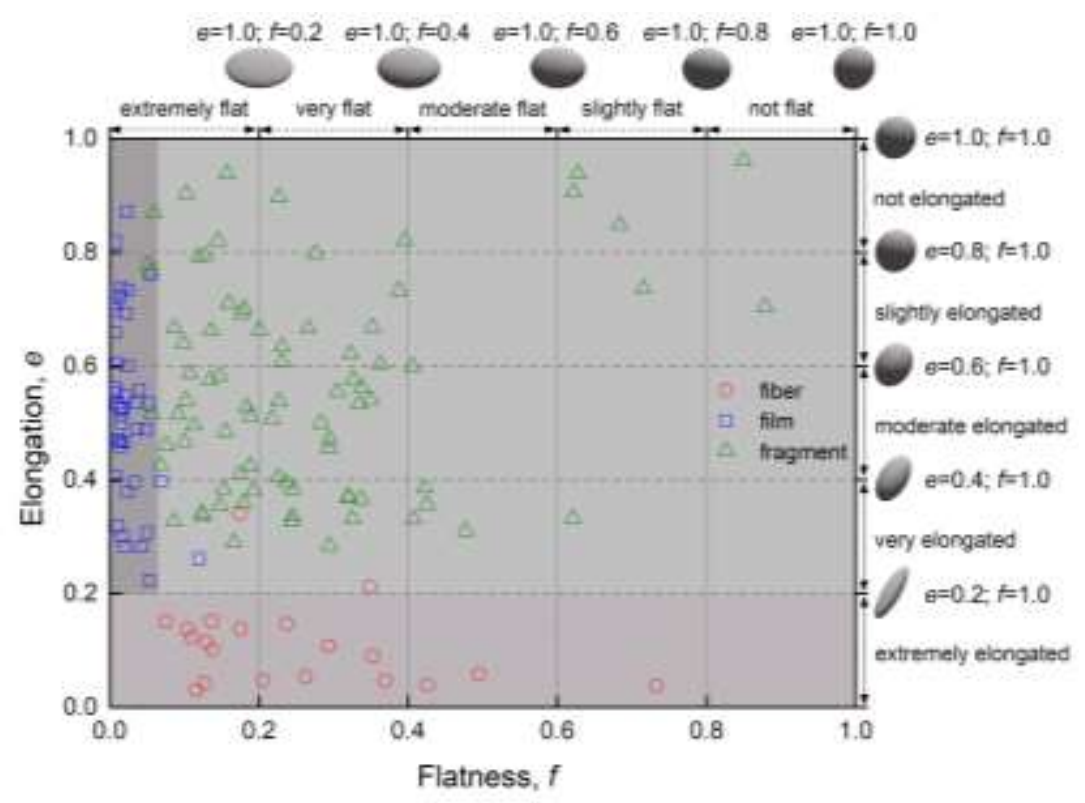

Figure S5. Microplastic classification based on elongation and flatness. The grid lines are plotted according to the shape classification framework of Blott et al. ${ }^{4}$. The ellipsoid that is widely used as a reference morphology in classification frameworks is adopted herein to serve as a reference.

It can be observed that the microplastic fragments fall into a wide range of shape regions. In contrast to the fragments, about $90 \%$ of the microplastic fibers fall within the extremely elongated region, and about $95 \%$ of microplastic films collapse into the region characterised as extremely flat. It is worthwhile to note that films or fragments do not fall into the extremely elongated region (i.e., $e<0.2$ ). Except for the extremely elongated region, the morphologies in other regions can somewhat be related to spheres using the projected area of a circle. This further supports the notion in our manuscript 
that the drag coefficient based on spheres in existing drag model is better suited for the prediction of the terminal settling velocity of microplastic fragments and films. These observations also corroborate that microplastic fibers are morphologically unique compared to microplastic fragments and films. Thus, research is prudent to improve the description of the settling motion of fibers.

While elongation appears to be best index to differentiate between fibrous and nonfibrous microplastics in terms of morphology, it is less suitable to differentiate fibers from fragments and films in terms of hydrodynamics. Thus, the hydrodynamic shape descriptor reported by Bagheri et al., ${ }^{5}$ specifically the Aschenbrenner shape factor, was evaluated in our manuscript on whether it can distinguish between the different microplastic morphologies. 


\section{SI4: Evaluation of Aschenbrenner shape factor with additional datasets}

The datasets reported by Khatmullina et al. ${ }^{1}$ and by Waldschlager et al. ${ }^{6}$ include fibrous and non-fibrous microplastics (MPs), respectively. Thus, these two datasets are combined to further evaluate the performance of the Aschenbrenner shape factor on distinguishing between fibrous and non-fibrous microplastics. Figure S6 compares the performance of the Aschenbrenner shape factor with the Corey shape factor on distinguishing fibrous and non-fibrous morphologies.

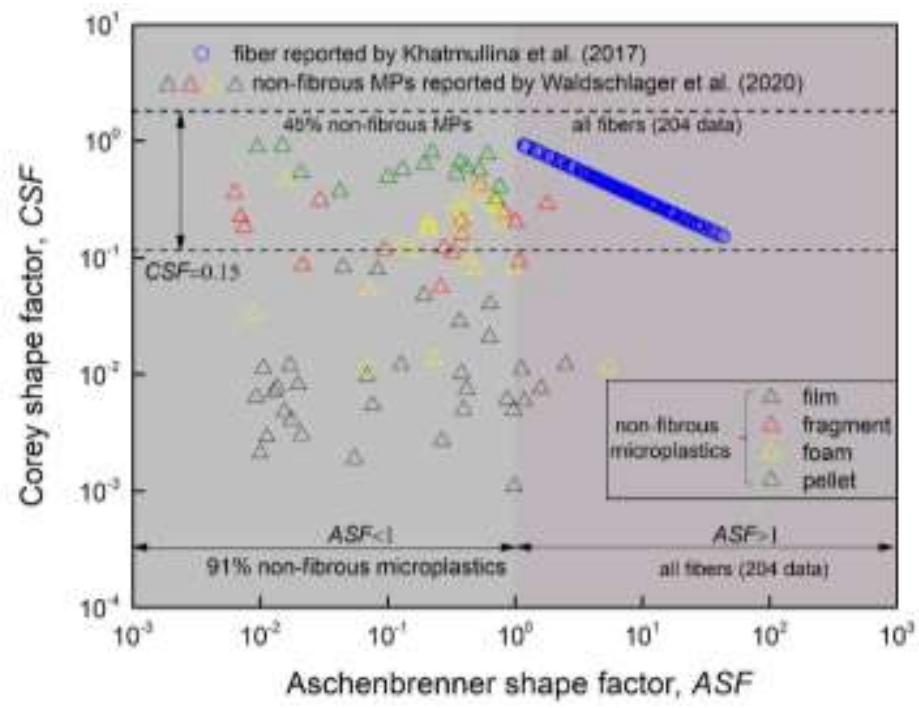

Figure S6. Comparison of performances of Aschenbrenner shape factor and Corey shape factors on distinguishing fibrous and non-fibrous microplastics.

It can be observed that $45 \%$ of non-fibrous microplastics (i.e., films, fragments, foams, and pellets) and all fibers have a similar range of Corey Shape factors, which is greater than 0.15. Compared with the Corey shape factor, the Aschenbrenner shape factor performs better at distinguishing between fibrous and non-fibrous morphologies. More specifically, all fibers have an Aschenbrenner shape factor that is larger than unity, while $91 \%$ of non-fibrous microplastics have an Aschenbrenner shape factor that is less 
than unity. Thus, it can be deduced that an $\mathrm{ASF}=1$ can serve as a criterion to distinguish between fibrous and non-fibrous microplastics. This criterion is consistent with the one that is drawn based on the data reported by Melkebeke et al. ${ }^{3}$.

Moreover, it should be noted that both the Aschenbrenner shape factor and the Corey shape factor are deficient at distinguishing among the films, fragments, foams, and pellets. This deficiency is mainly attributed to the fact that all of the non-fibrous microplastics reported by Waldschlager et al. ${ }^{5}$ are weathered microplastics collected from a fluvial environment instead of the virgin microplastics used in labs (i.e., the microplastics reported by Khatmullina et al. ${ }^{1}$ and Melkebeke et al. ${ }^{3}$ ). Thus far, there is a dearth of knowledge on how weathering process (e.g., fragmentation, degradation, and biofouling) influences the morphologies of the microplastics in the field. Therefore, it is unclear why the Aschenbrenner shape factor fails to differentiate between the morphologies of weathered non-fibrous microplastics (i.e., films, fragments, foams, and pellets).

Another interesting result is that the Corey shape factors of the fibers reported by Khatmullina et al. ${ }^{1}$ vary linearly with the Aschenbrenner shape factors (see Figure S6). This observed trend is mainly due to the cylindrical morphology of the fibers used by Khatmullina et al. ${ }^{1}$ More specifically, the Corey shape factors show the same morphological meaning as the Aschenbrenner shape factor, which has been discussed previously. 


\section{SI5: Assessment of extracted data}

The sizes and terminal settling velocities of microplastic fibers (204 data points) were extracted from the experiment conducted by Khatmullina et al. ${ }^{1}$ to evaluate the newlyproposed drag model (Eq.6). The extracted data points are assessed in terms of extraction error. More specifically, with the data extracted from Khatmullina et al. ${ }^{1}$, we first recalculated the terminal settling velocities of fibers by using the drag model proposed by Khatmullina et al. ${ }^{1}$ Subsequently, we recalculated the average error $(A E)$ and the coefficient of determination $\left(R^{2}\right)$ by comparing the recalculated settling velocities and the extracted (i.e., measured) settling velocities. Finally, we compared the prediction accuracy (i.e., $A E$ and $R^{2}$ ) reported by Khatmullina et al. ${ }^{1}$ and the ones recalculated by using the extracted data, as summarised in Table S3.

Table S3 Comparison between reported and recalculated prediction accuracy

\begin{tabular}{ccccc}
\hline \multirow{2}{*}{ Fiber diameter } & \multicolumn{2}{c}{ Reported by Khatmullina et al. ${ }^{1}$} & \multicolumn{2}{c}{ Recalculated by using extracted data } \\
\cline { 2 - 5 } & $A E$ & $R^{2}$ & $A E$ & $R^{2}$ \\
\hline $0.15 \mathrm{~mm}$ & $10.10 \%$ & 0.807 & $10.19 \%$ & 0.796 \\
$0.22 \mathrm{~mm}$ & $6.50 \%$ & 0.917 & $6.41 \%$ & 0.906 \\
$0.34 \mathrm{~mm}$ & $10.90 \%$ & 0.949 & $10.98 \%$ & 0.946 \\
$0.46 \mathrm{~mm}$ & $2.30 \%$ & 0.965 & $2.69 \%$ & 0.973 \\
$0.60 \mathrm{~mm}$ & $5.00 \%$ & 0.953 & $4.94 \%$ & 0.948 \\
$0.71 \mathrm{~mm}$ & $2.70 \%$ & 0.906 & $2.73 \%$ & 0.908 \\
All fibers & $6.00 \%$ & 0.982 & $6.49 \%$ & 0.987 \\
\hline
\end{tabular}

For all groups of fibers, the differences between the average errors (AE) and the coefficients of determination $\left(\mathrm{R}^{2}\right)$ are less than $0.5 \%$ and 0.015 , respectively. The results show the high reliability of the extracted data. 
SI6: Evaluation of new drag model with estimated fiber volume based on the experiment data produced in this study

Based on Figure 4 in our manuscript, Figure S7 shows the calculated terminal settling velocities by using the newly-proposed drag model and estimated fiber volumes $\left(V^{\prime}=\right.$ $\left.D_{\mathrm{L}} D_{\mathrm{I}} D_{\mathrm{S}}\right)$. It can be observed that, even with the estimated fiber volumes, the newlyproposed drag model (Eq.6) still exhibits a comparatively improved performance in predictions of the terminal settling velocities of microplastic fibers compared with the models proposed by Khatmullina et al. ${ }^{1}$, Dioguardi et al. ${ }^{7}$, and Waldschlager et al. ${ }^{2}$

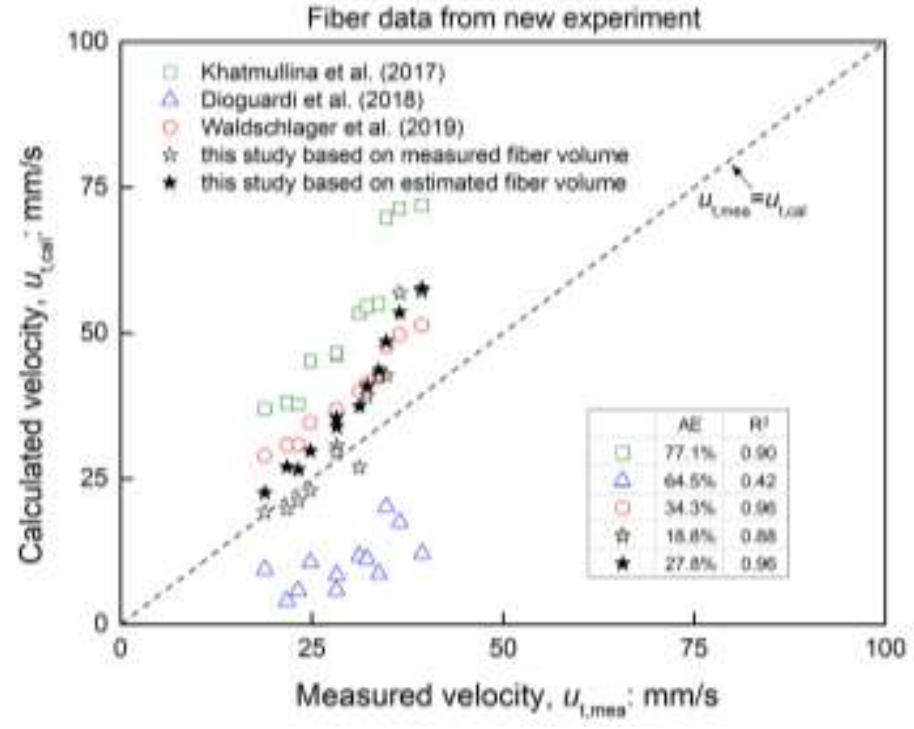

Figure S7. Evaluation of newly-proposed model (Eq.6) with the estimated fiber volume provided by experiments conducted in this study. 


\section{SI7: Applicability of new drag model to weathered microplastics}

Based on the dataset of weathered microplastics reported by Waldschlager et al..$^{5}$, the performance of the new drag model proposed by this study and the model proposed by Waldschlager et $\mathrm{al}^{2}$ on predicting the terminal settling and rising velocities of weathered microplastics are evaluated in Figure S8.

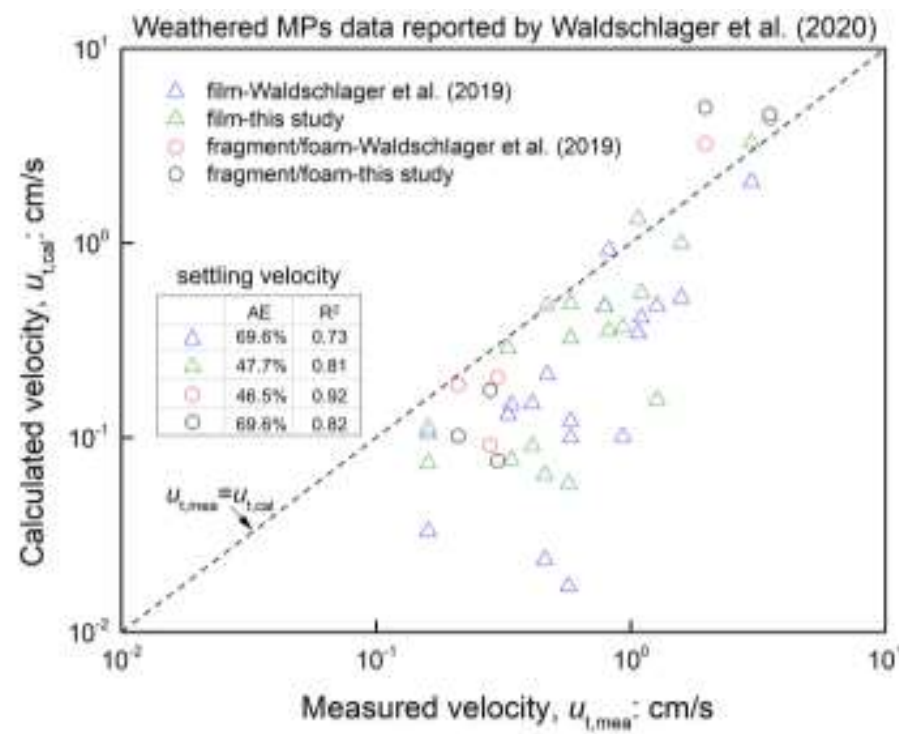

(a)

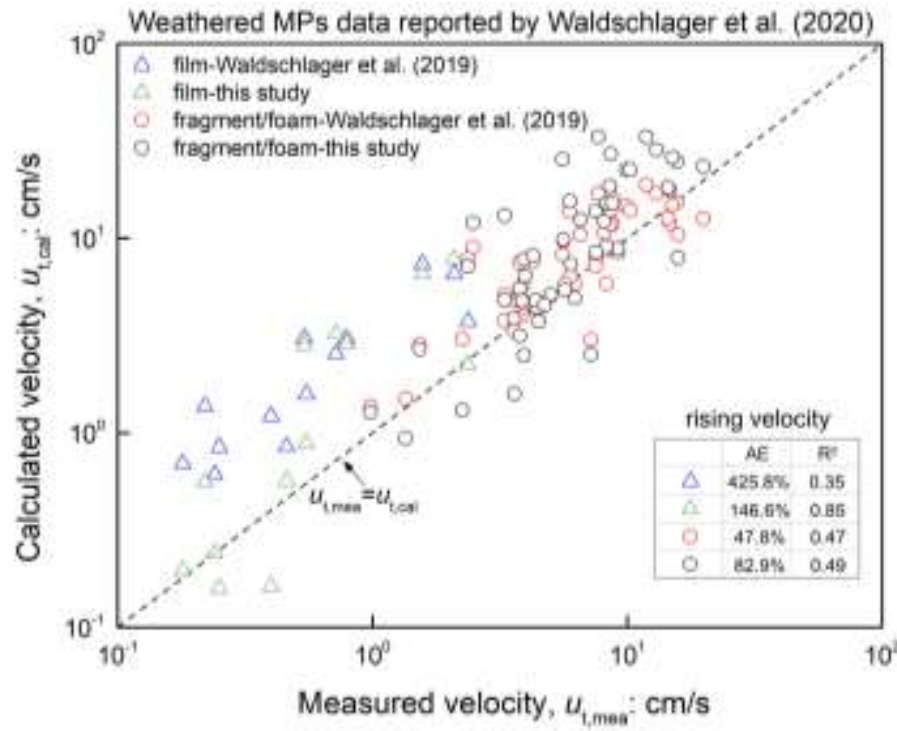

(b)

Figure S8. Comparison of performances of drag models proposed by this study and by Waldschlager et al. ${ }^{2}$ on predicting terminal settling/rising velocities of weathered microplastics.

(a) Terminal settling velocities; (b) terminal rising velocities. 
It can be found from Figure S8a that the two models exhibit similar performances on predictions of the terminal settling velocities of weathered microplastics. More specifically, both models underestimate the terminal settling velocities of weathered microplastics with average errors of $46.5 \% \sim 69.6 \%$.

In Figure S8b, the model proposed by Waldschlager et al. ${ }^{2}$ exhibits reasonable performance for predictions of the terminal rising velocities of weathered fragments and foams $\left(\mathrm{AE}=47.8 \% \mathrm{R}^{2}=0.47\right)$ compared with the measured velocities. However, the predictions of the terminal rising velocities for weathered films do not show reasonable agreement with the measured ones $\left(\mathrm{AE}=425.8 \% \mathrm{R}^{2}=0.35\right)$. By comparison, the newlyproposed model in this study does not perform as well as that proposed by Waldschlager et al. ${ }^{2}$ for predicting the rising velocities of fragments and foams $\left(\mathrm{AE}=82.9 \% \mathrm{R}^{2}=0.49\right)$. However, the newly-proposed drag model exhibits improved predictions of the terminal rising velocities for weathered films $\left(\mathrm{AE}=146.6 \% \mathrm{R}^{2}=0.85\right)$ compared to that proposed by Waldschlager et al. $^{2}$. Nonetheless, it can be concluded that existing models are generally deficient at reasonably predicting the terminal rising velocities of weathered films. 


\section{SI8: Applicability of new drag model to predictions of terminal rising velocities of}

\section{virgin microplastics}

Waldschlager et al. $^{2}$ reported a dataset of terminal rising velocities for virgin microplastics (MPs), including fibers, fragments, and pellets. Based on this dataset, the performance of the newly-proposed drag model on predicting the terminal rising velocities of microplastics are evaluated in Figure S9. Also, the performance of the model proposed by using this dataset, which has been reported in the literature, ${ }^{2}$ serve herein as the reference for the evaluation of the newly-drag model.

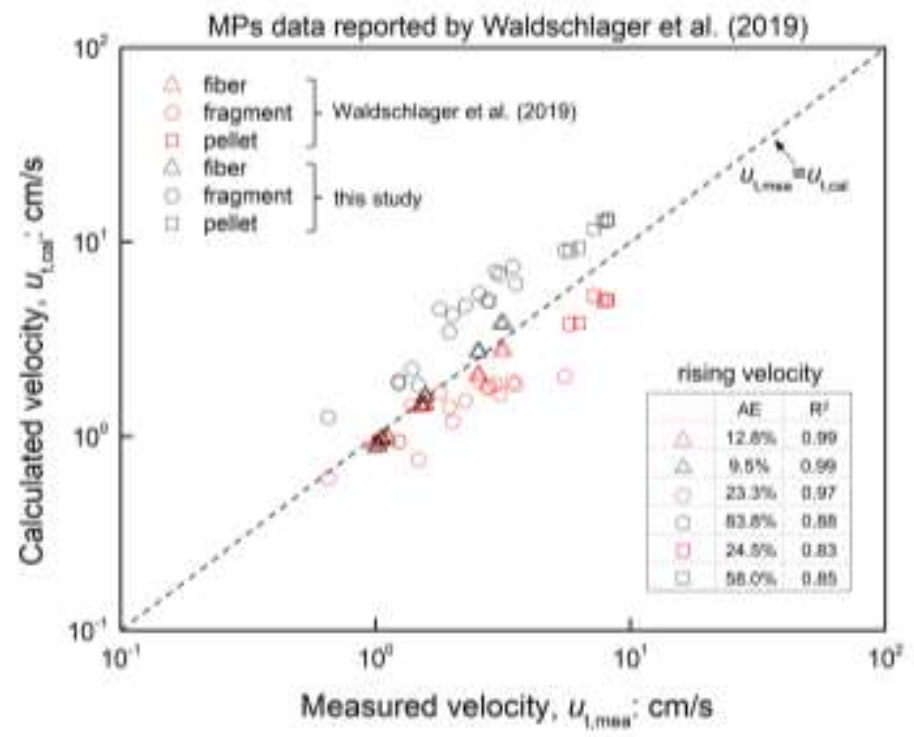

Figure S9. Performances of drag models proposed by this study and by Waldschlager et al. ${ }^{2}$ on predicting terminal rising velocities of virgin microplastics.

It can be observed that, similarly to the model proposed by Waldschlager et al. ${ }^{2}$ ( $A E=12.8 \%$ and $R^{2}=0.99$ ), the newly-proposed drag model can also reasonably predict the terminal rising velocities of microplastic fibers $\left(A E=9.5 \%\right.$ and $\left.R^{2}=0.99\right)$. This result is not surprising because the underlying principles between the settling and rising 
motion of fibers do not differ significantly. Based on the dataset used in Figure S9, Waldschlager et al. $^{2}$ proposed the following drag models for the predictions of the settling and rising velocities of fibers:

For settling fibers:

$C_{D}=\frac{4.7}{\sqrt{R e}}+\sqrt{C S F}$

For rising fibers:

$C_{D}=\frac{10}{\sqrt{R e}}+\sqrt{C S F}$

Evidently, these two expressions are similar in form. Thus, the newly-proposed drag model, which has been shown to be able to reasonably predict the settling velocities of fibers, should enable reasonable predictions of the rising velocities of fibers.

In contrast with fibers, the newly-proposed drag model overestimates the terminal rising velocities of fragments and pellets with an average error of $83.8 \%$ and $58.0 \%$, respectively. The over-predictions may be because the dataset reported by Waldschlager et al. $^{2}$ does not provide information on the fiber volume, and thus, the estimated volumes based on fiber lengths were adopted herein to evaluate the newly-proposed model in this study. Moreover, the settling expression proposed by Waldschlager et al. ${ }^{2}$ for non-fibrous microplastics is significantly different from their rising ones, as shown 
below:

For settling non-fibrous microplastics:

$$
C_{D}=\frac{3}{C S F \times \sqrt[3]{R e}}
$$

For rising non-fibrous microplastics:

$$
C_{D}=\left(\frac{20}{R e}+\frac{10}{\sqrt{R e}}+\sqrt{1.195-C S F}\right) \times\left(\frac{6}{P}\right)^{1-C S F}
$$

It can be inferred that the rising motion of non-fibrous microplastics significantly differs from their settling motion since the rising expression (Eq.S2-2) is noticeably different from the setting one (Eq.S2-1). Thus, it may be understandable that the newlyproposed model, which was developed based on data of the settling motion of microplastics may not reasonably be able to predict the rising velocities of non-fibrous microplastics. 


\section{References}

1. Khatmullina, L.; Isachenko, I. Settling velocity of microplastic particles of regular shapes. Mar. Pollut. Bull. 2017, 114, 871-880.

2. Waldschlager, K.; Schuttrumpf, H. Effects of particle properties on the settling and rise velocities of microplastics in freshwater under laboratory conditions. Environ. Sci. Technol. 2019, 53, 1958-1966.

3. Melkebeke, M. V.; Janssen, C.; De Meester, S. Characteristics and sinking behavior of typical microplastics including the potential effect of biofouling: Implications for remediation. Environ. Sci. Technol. 2020, 54, 8668-8680.

4. Blott, S. J.; Pye, K. Particle shape: A review and new methods of characterization and classification. Sedimentology 2008, 55, 31-63.

5. Bagheri, G.; Bonadonna, C. On the drag of freely falling non-spherical particles. Powder Technol. 2016, 301, 526-544.

6. Waldschlager, K.; Born, M.; Cowger, W.; Gray, A.; Schuttrumpf, H. Settling and rising velocities of environmentally weathered micro- and macroplastic particles. Environ. Res. 2020, 191, 110192.

7. Dioguardi, F.; Mele, D.; Dellino, P. A new one-equation model of fluid drag for irregularly shaped particles valid over a wide range of Reynolds number. J. Geophys. Res.: Solid Earth 2018, 123, 144-156. 